ISSN: 1130-2887 - e-ISSN: 2340-4396

\title{
PRESENTACIÓN. POLÍTICA, ESPACIALIDAD Y TERRITORIALIDAD. HACIA UN ANÁLISIS ESPACIAL DE LOS PROCESOS SOCIO-POLÍTICOS EN AMÉRICA LATINA
}

Hoy día existe una profusión de mapas en las redes sociales y en los medios de comunicación. La generalización de la geolocalización y de la cartografía permite ubicar dónde comemos y trabajamos, nos divertimos o fotografiamos, por qué medio y hacia dónde nos desplazamos, al grado de transformar la geografía en una construcción virtual y trivial. No obstante, a pesar del incremento exponencial de la información georreferenciada, todavía carecemos de una teoría unificada para entender las modalidades y funciones que desempeña el espacio en el desarrollo de los procesos políticos, sociales, económicos y culturales. ¿Para qué sirven los enfoques territoriales? ¿Por qué el espacio importa en las ciencias sociales? ¿Cuál es la trayectoria del análisis espacial y cuáles son sus perspectivas en América Latina?

\section{TRAYECTORIA Y POTENCIAL TEÓRICO-METODOLÓGICO DEL ANÁLISIS ESPACIAL}

El análisis espacial busca situar a los actores dentro de contextos y entornos más amplios, que posibilitan y constriñen la agencia humana. Más allá de sus características psicológicas individuales, el ser humano también es un animal social y territorial, cuyo comportamiento depende del pueblo o barrio en el que reside, de la escuela en la que ha sido educado y del lugar en el que trabaja, de los vecinos que frecuenta y de las redes de sociabilidad que comparte. Por ello, importa observar los comportamientos individuales en el contexto concreto, particular y situado en el que se produce y se desarrolla la interacción social.

Este enfoque holístico e integral fue fundamental para sentar las bases de las ciencias sociales. Las investigaciones seminales que fundaron el estudio científico de las epidemias y de otras cuestiones de salud pública; de la criminalidad y de otras desviaciones sociales; de la pobreza, la desigualdad y otras formas de exclusión social; del voto y de las geografías electorales; todas ellas desconocían las rencillas contemporáneas entre cuantitativistas y cualitativistas y se enfocaban en los problemas fundamentales: ¿Cómo se difunde el cólera y qué medidas pueden tomarse para contrarrestarlo? ¿Cómo se reproducen la pobreza y la desigualdad? ¿Qué papel juega la etnicidad en los procesos 
de migración y de urbanización? ¿Por qué el voto es tan coherente y estable, cuando la política es tan caótica y volátil?

Estos estudios magistrales desarrollados por pioneros como John Snow en 1854 (RSPH 2017), Charles Booth (LSE 2017), Florence Kelley (NorthwesternLaw 2017) o André Siegfried (1913) anticiparon e inspiraron el desarrollo ulterior de un sinfín de técnicas y métodos, de observación directa y etnográfica, de estudios de casos densos y a profundidad, de muestreos aleatorios probabilísticos y de modelos de regresión estadística cada vez más sofisticados. Entre otras vertientes, el estudio espacial e integral de unidades territoriales situadas alcanzó sus títulos de nobleza con el éxito de la Escuela de Chicago de sociología y de ecología urbana (Park y Burgess 1921). La estrategia analítica consistía en observar a los individuos en sus hábitats para explicar cómo el comportamiento agregado podía revelar patrones de interacción colectiva y estructuras sociales estables. Entre sus objetos privilegiados de estudio se encuentran la segregación urbana, la criminalidad o la desigualdad plasmada en el espacio.

La geografía electoral merece una mención especial, porque permite ilustrar la suerte cambiante del análisis espacial a la luz del desarrollo histórico del estudio académico del voto. Desde el estudio pionero de Siegfried (1913) en Francia, esta disciplina se ha ocupado de entender cómo factores demográficos, económicos y relacionados a la geografía humana (densidad demográfica, ocupación del suelo, tipo de propiedad agraria, etc.) se relacionan con el comportamiento electoral y la representación política. Otros se concentraron en saber cómo se delimitaban las circunscripciones y cómo se convertían los votos en escaños, dado que siempre se requiere de alguna regla de agregación espacial para lograr tal objetivo.

No obstante, después de haber desempeñado un papel crucial en la fundación de la ciencia política moderna, el estudio territorial del voto fue desplazado por el desarrollo de las encuestas de opinión en la década de 1940, de los enfoques sicosociales en los cincuenta y del paradigma de la elección racional en los setenta, antes de resurgir bajo una forma renovada en los noventa, redescubierta y enriquecida por la nueva geografía política y humana. El enfoque «ecológico» del comportamiento electoral sufrió así los embates del desarrollo y del auge de las encuestas «individualistas» de opinión pública que, en su búsqueda de modelos generales y universales, desestimaron las especificidades territoriales y los efectos contextuales para privilegiar el estudio de las actitudes, las convicciones, las conductas, los cálculos y las preferencias personales de los electores.

A partir de entonces, los principales modelos explicativos del voto lo analizaron como un conjunto fuertemente integrado de preferencias sicosociales y de elecciones individuales, asumiendo que los electorados están suficientemente integrados para ser analizados bajo un mismo esquema unificado, como un simple agregado de actitudes e identidades, orientaciones y preferencias individuales. En esta óptica, el voto es concebido como una respuesta individual unívoca a una pregunta universal inequívoca, ya que se asume que ambas tienen un sentido común que permite agregarlas independientemente de la diversidad de significados que pueden conferirles las especificidades sociales, territoriales, culturales y situacionales (Sonnleitner 2013). 
La difusión y el éxito de estos métodos fue tal que, no solamente se impusieron como el enfoque privilegiado para la interpretación y la explicación del voto, sino que hasta marginaron los análisis ecológicos de la ciencia política. Al prescindir de toda reflexión sobre la dimensión territorial del voto, el enfoque geográfico sucumbió a la hegemonía de una sociología electoral sin perspectiva espacial.

Entre las razones que explican este cambio de paradigma, cabe destacar la crítica del determinismo geográfico con el que se pudo asociar al análisis ecológico, en un contexto histórico en el que la urbanización, el desarrollo de los medios masivos de comunicación e impresionantes avances de la integración nacional subrayaban la homogeneización de las sociedades, haciendo pasar a un segundo plano su diversidad, sus divisiones y sus particularismos internos (Bussi 1998).

Además de fundamentarse en un método reputado como «fastidioso»-la elaboración manual de un mapa exigía entonces muchísimo tiempo y dedicación (Goguel 1990)-, la geografía electoral también sufrió otra crítica simplificadora relacionada con la famosa «falacia ecológica» (ecological fallacy). El cuestionamiento radical del análisis de datos agregados, planteado por W. S. Robinson (1950) en pleno auge del individualismo metodológico, fue tan influyente que generó una suerte de dogma en las ciencias sociales hegemónicas, relegando el enfoque territorial a los guetos acotados de los estudios urbanos y de la geografía electoral ${ }^{1}$.

Desde entonces, este argumento fue (y sigue siendo) utilizado recurrentemente para descalificar la validez de los análisis ecológicos. No obstante, se trata de una interpretación reductora de un fenómeno más complejo que, lejos de invalidar la utilidad del enfoque territorial, confirma precisamente su necesidad. Ciertamente, la relación estadística entre dos fenómenos sociopolíticos cambia cuando se modifica el nivel de observación, disminuyendo generalmente su intensidad al incrementarse las unidades de análisis, e invirtiendo incluso en ocasiones su signo matemático. Asimismo, las correlaciones que pueden establecerse entre unidades colectivas dependen inevitablemente de los criterios de delimitación y agregación que se utilizan para construir dichas unidades, lo que también se conoce como el Problema de Unidades de Área Modificables (Modifiable Areal Unit Problem, MAUP).

Sin embargo, para poder superar la falacia «ecológica» sin caer en la trampa inversa de reducir toda conducta social a una mera suma de comportamientos individuales e independientes (la llamada falacia «atomística»), resulta indispensable reflexionar sobre las razones y los significados empíricos de las correlaciones cambiantes que se producen en los distintos niveles de observación entre las distintas unidades de análisis, lo que exige adoptar enfoques multidimensionales que permitan articular las distintas escalas analíticas.

1. Al analizar la correlación entre el analfabetismo y la proporción de ciudadanos afroamericanos en distintas escalas de la geografía estadounidense, W. S. Robinson advirtió que ésta era muy fuerte en el nivel de las nueve divisiones geográficas $(+0,946)$, pero se reducía en el nivel de los 48 estados $(+0,773)$ para alcanzar tan sólo $+0,203$ en el nivel de los 97,3 millones de individuos mayores a diez años censados en 1930. De ello, concluyó que había que privilegiar las correlaciones individuales en lugar de computar correlaciones colectivas «sin sentido» (W. ROBINSON 1950: 353-357). 
Solo al final de los años 1960 y, posteriormente, con la revolución informática, se ha podido recuperar una reflexión más sistemática sobre el papel del espacio en las ciencias sociales. Algunos autores como Goodchild y Janelle $(2004,2010)$ o Logan (2012) destacan el desarrollo metodológico de los estudios espaciales al mismo tiempo que apuntan hacia la incipiente capacidad analítica y crítica de los métodos que recurren a la geoestadística, los Sistemas de Información Geográfica (SIG) o la econometría espacial (Anselin 1988).

Hubo que esperar, así, los cambios tecnológicos, teóricos y epistemológicos de los ochenta para que resurgiera con fuerza, y en una forma renovada, la geografía electoral. Con la crisis del Estado-Nación, los regionalismos y las tradiciones locales se reafirmaron con vehemencia. Ello suscitó un interés creciente por las representaciones e identidades políticas territorializadas entre muchos antropólogos y geógrafos, quienes descubrieron la cantidad y la calidad extraordinarias de los resultados electorales, disponibles en todas las escalas deseables e imaginables. Beneficiándose del desarrollo de las bases de datos informáticas, de la cartografía automatizada y de SIG cada vez más sofisticados, una nueva generación de estudios, realizados en un primer tiempo por investigadores externos a la Ciencia Política, retomó la tarea de explorar las dimensiones espaciales del voto².

Finalmente, como consecuencia de las políticas de descentralización, de la multiplicación y del desarrollo de elecciones locales, la llamada «nacionalización» de los comportamientos electorales, que se había acompañado de la reducción de las especificidades territoriales, empezó a debilitarse. Y la afirmación de nuevas fuerzas políticoelectorales fuertemente regionalizadas, que vinieron a llenar los espacios dejados por el reflujo y la fragmentación de los partidos tradicionales de masa, incitó a los politólogos a reconciliarse con los padres fundadores y a redescubrir la dimensión territorial de los comportamientos electorales (Bussi 1998).

Hoy en día, se reconoce que los enfoques ecológicos e individuales son compatibles, y que pueden ser combinados mediante aproximaciones mixtas (Mayer y Perrineau 1992). En efecto, se pueden analizar tanto datos individuales agregados para caracterizar las unidades territoriales analizadas, como datos colectivos derivados de los lugares de residencia para saber más sobre el contexto habitacional de los individuos estudiados. En resumidas cuentas, el análisis multidimensional del voto puede y debe enriquecerse de ambos enfoques teórico-metodológicos, a condición de tener presentes sus respectivos alcances y limitaciones.

\section{Perspectivas latinoamericanas}

En América Latina, se han realizado numerosas investigaciones de carácter monográfico. Éstas se centran en el estudio de elecciones particulares en países y regiones

2. Entre ellos, cabe destacar los trabajos de: F. Goguel (1981-1983), H. LE BrAS y E. TODD (1981), Y. Lacoste (1986), F. Bon y J. P. Cheylan (1988), M. Bussi (1998), H. Le Bras (2002) y P. Waniez (2002 y 2008) en Francia; así como los trabajos de K. Cox (1969), R. JoHnston (1979), P. TAylor y J. House (1984), J. Agnew (1996) y L. Anselin (1988) en Inglaterra y los Ee. uU. 
específicas, enfocándose generalmente en la descripción de la distribución territorial y en la explicación ecológica del voto. Inspiradas por las escuelas francesa y anglosajonas, y bajo el impulso de los estudios realizados/coordinados por Juan Molinar Horcasitas (1991), Gustavo Ernesto Emmerich (1993) y Silvia Gómez Tagle y María Eugenia Valdés (2000), se está desarrollado una importante corriente de trabajos de este tipo en México. Al respecto, cabe citar las decenas de ponencias presentadas en los congresos anuales de la Sociedad Mexicana de Estudios Electorales (SOMEE), que cuenta ocasionalmente con ejes temáticos dedicados expresamente a la geografía electoral’3.

Asimismo, se han estado configurando una serie de proyectos e iniciativas de cooperación científica e investigación académica colectiva que reflejan el interés creciente por el análisis espacial del voto en la región. Entre 2001 y 2004, el Institut des Hautes Études de l'Amérique Latine (IHEAL) de la Universidad de París III impulsó un proyecto pionero intitulado «Atlas Electoral de América Latina», en el que participó un nutrido grupo de latinoamericanistas. En ese marco, se organizaron seis seminarios-coloquios internacionales en Bogotá (2002), Biarritz (2002), Lima (2003) y París (2001, 2002 y 2003), que permitieron ricos intercambios académicos y se materializaron en dos trabajos colectivos ${ }^{4}$.

En noviembre de 2006, la Corte Nacional Electoral (CNE) de Bolivia le dio continuidad a dicha iniciativa, reunió a un grupo de latinoamericanistas en las orillas del lago Titicaca y patrocinó una tercera publicación colectiva (Romero Ballivian 2007)5. A su vez, muchos de los investigadores asociados a esta iniciativa publicaron sus propios trabajos individuales sobre diversos países de la región, alimentando una creciente literatura sobre la geografía electoral de América Latina ${ }^{6}$.

Entre 2004 y 2007, se desarrolló otro proyecto colectivo de geografía electoral en Centroamérica, gracias a los auspicios del Centro francés de Estudios Mexicanos y Centroamericanos (CEMCA), del Banco Interamericano de Desarrollo (BID) y del IHEAL. Éste permitió la realización de siete seminarios-talleres de cartografía electoral: Tegucigalpa, Managua, San

3. Como botón de muestra, durante el XXIII Congreso Nacional de Estudios Electorales: Partidos y Elecciones en la Disputa Nacional, realizado del 31 de octubre al 2 de noviembre de 2012, se presentaron ocho contribuciones en el marco del eje temático «Geografía Electoral».

4. En este proyecto participaron: Yann Basset (para Argentina); Salvador Romero Ballivián (para Bolivia); Cesar Romero Jacob, Dora Rodrigues Hees, Philippe Waniez y Violette Brustlein (para Brasil); Fernando Giraldo, Rodrigo Losada y Patricia Muñoz (para Colombia); Simón Pachano (para Ecuador); Fernando Tuesta y Jorge Valladares (para Perú); Tibisay Lucena y Carmen Pérez Baralt (para Venezuela); Alain de Remes (para México); así como Jean-Michel Blanquer y Willibald Sonnleitner (quienes elaboraron trabajos sobre Colombia y México, y coordinaron el proyecto). Parte de los resultados de esta investigación se publicaron bajo la forma de dos trabajos colectivos (J-M. BLANQUER, F. GIRALDO y W. Sonnleitner 2003; J.-M. Blanquer, H. Quanquin, W. Sonnleitner y C. Zumello 2005).

5. En esta iniciativa participaron, además de las personas ya mencionadas, Adriana Castro (Colombia), Hugo Picado León (Costa Rica), Carlos Vargas León (Perú), Stéphanie Alenda y Georges Couffignal (Chile).

6. Al respecto, cabe citar los trabajos de P. Waniez, V. Brustlein, C. Jacob y D. Hees (2000, 2008); S. Romero Ballivián (2003); R. Losada, F. Giraldo y P. Muñoz (2004); C. Villalta PerDOMO (2006); Y. BASSET (2011). 
Salvador, San José de Costa Rica y Guatemala-Ciudad en 2004, antes de materializarse en dos publicaciones colectivas que contienen los resultados de dicho esfuerzo de cooperación científica (Sonnleitner 2005 y 2006).

En el Cono Sur cabe destacar, además de los trabajos ya citados de Cesar Romero Jacob, Dora Rodrigues Hees, Violette Brustlein y Philippe Waniez (2006 y 2010), los estudios impulsados por Sonia Terrón y Glaucio Soares desde el Instituto Universitário de Pesquisas do Rio de Janeiro (IUPERJ) y el Instituto de Estudos Sociais e Políticos (IESP) de la Universidad Estatal de Rio de Janeiro en Brasil (Soares y Terron 2008; Terron 2009; Terron y Soares 2010). Cabe destacar a su vez la realización del I Seminário Internacional Instituições, Comportamento Político e Geografia do Voto (GEOvOTO) en la Universidade de Brasilia, en 2012, bajo la coordinación de André Borges. En Argentina las investigaciones realizadas bajo la iniciativa de Marcelo Escolar desde el Centro de Estudios Federales y Electorales (CEFE), de la Escuela de Política y Gobierno de la Universidad Nacional de San Martín (UNSAM), también han hecho importantes contribuciones a la geografía electoral latinoamericanista (Calvo y Escolar 2003; Escolar y Calvo 2005; Escolar y Castro 2012).

En resumidas cuentas, todas estas iniciativas e investigaciones, colectivas e individuales, evidencian el interés creciente por el análisis territorial y espacial, que está abriendo nuevas vetas de estudio y reconfigurando las agendas de investigación en América Latina. Ello invita a reflexionar sobre las posibilidades concretas que ofrecen la cartografía y el análisis exploratorio de datos espaciales como herramientas teórico-metodológicas y analíticas. Invita, asimismo, a realizar un balance más amplio, completo y actualizado de la utilización de métodos espaciales en la investigación sociopolítica latinoamericanista, que incluya otros proyectos e iniciativas que se están desarrollando sobre políticas públicas, criminalidad, violencia e inseguridad.

Para facilitar la confluencia y el diálogo entre todas estas agendas de investigación se ha formado un grupo de trabajo dedicado al análisis espacial dentro de la Asociación Latinoamericana de Ciencia Política (ALACIP). Denominado Espacio Alacip, fue creado en 2011 y no ha dejado de crecer desde entonces, reuniendo ahora a más de 40 investigadores interesados en el análisis territorial del voto, provenientes por lo pronto de siete países de Latinoamérica ${ }^{7}$. Sumándose a los esfuerzos de Espacio Alacip, el Laboratorio de análisis espacial aplicado a las ciencias sociales (CompassLab), fundado por Rodrigo Rodrigues da Silveira, también impulsa el análisis espacial desde la Unversidad de Salamanca ${ }^{8}$. En 2016, unimos esfuerzos para coordinar el Simposio metodológico «Política sub-nacional, espacialidades globales y territorialidades transnacionales: enfoques y perspectivas de análisis desde Europa y América Latina», en el marco del Congreso Internacional del Consejo Europeo de Investigaciones y Ciencias Sociales sobre América Latina (CEISAL), en cuyo marco

7. Para mayor información sobre el Grupo de Investigación en Análisis Espacial en América Latina de la Asociación Latinoamericana de Ciencia Política (Espacio ALACIP), véase: http://espacioalacip.net/.

8. Para mayor información sobre CompassLab, véase: http://www.compasslab.org. 
se elaboraron y debatieron las contribuciones de este volumen especial de análisis espacial de América Latina Hoy, Revista de Ciencias Sociales.

\section{POLÍTICA SUBNACIONAL, ESPACIALIDADES Y TERRITORIALIDADES}

El dosier temático de este volumen reúne cuatro artículos con perspectivas diversas de análisis en las que el espacio ocupa un lugar central. La investigación de Willibald Sonnleitner indaga en las dinámicas recientes de la descomposición del sistema de partidos en México. Más allá de las pugnas intra-partidistas coyunturales, éstas obedecen a pautas temporales y territoriales fuertemente estructuradas que se remontan al menos a la década de los noventa. Las raíces socioterritoriales de esta profunda transformación aparecen con claridad en el nivel de los 300 distritos en los que se eligen los diputados federales uninominales. La distribución geográfica y el perfil sociodemográfico de la fragmentación permiten detectar sus orígenes y ubicar la presencia desigual de los viejos y nuevos actores que conforman la geografía político-electoral mexicana, en una perspectiva subnacional que no sería inteligible sin el uso de métodos espaciales.

Emerson Urizzi Cervi revisita, a partir de un abordaje novedoso, el debate sobre las bases geográficas cambiantes del Partido de los Trabajadores (PT) y del Partido de la Social Democracia Brasileña (PSDB). Se centra en las dos fuerzas que polarizaron las elecciones presidenciales desde 1994, y avanza en la búsqueda de nuevas hipótesis para explicar estos cambios desde la perspectiva de los principales partidos de gobierno y de oposición en Brasil. Más allá de su contribución al debate político-partidista, constituye un aporte interesante al conjunto de estudios recientes sobre la nueva geografía electoral brasileña, haciendo un uso imaginativo de distintos métodos de análisis espacial.

En perspectiva comparada, Evelise Z. da Silva, Samira Kauchakje y Luciene S. Delazari analizan en qué formas la difusión de las ideas promovidas por los organismos económicos internacionales puede haber influido en la toma de decisiones de los gobiernos de América Latina y el Caribe, al fomentar la adopción de políticas de Conditional Cash Transfer con similitudes institucionales significativas. La espacialidad y territorialidad son herramientas cruciales para esta investigación, que utiliza métodos cualitativos y mapas para analizar la interferencia de la escala global sobre la nacional, independientemente de las disparidades en las trayectorias políticas, económicas y sociales de los países estudiados.

Finalmente, Mariana Schweitzer investiga el impacto local de otros procesos globales a partir del caso de la Hidrovía Paraná-Paraguay, en el que los territorios de Puerto General San Martín y San Lorenzo han recibido inversiones multimillonarias para el desarrollo de terminales portuarias destinadas a la exportación de commodities. Su análisis espacio-temporal de las transformaciones locales en comparación con las ocurridas a nivel provincial y nacional, a través de indicadores sociodemográficos, económicos y geoambientales, revela impactos negativos y positivos, además de tensiones y conflictos generados por los intereses contrapuestos de las empresas y la población local, con la intermediación de gobiernos locales débiles y comprometidos con los intereses del capital. 
Por otra parte, se incluyen dos artículos adicionales en la sección VARIA. El primero, de Víctor Carlos Pascual Planchuelo, examina las últimas elecciones en Venezuela y se enfoca en la participación de la OEA y de la UNASUR. Con esta base empírica, identifica y distingue dos modalidades distintas, de «observación» y de «acompañamiento» electoral. En el segundo artículo, Germán Albuquerque analiza la política exterior del régimen militar de la década de 1970 en Perú. Ésta se ubica en un nuevo paradigma que se difundió en toda América Latina, en el cual la ideología tercermundista fue reapropiada y reelaborada para estos fines específicos.

El presente esfuerzo colectivo ofrece, en suma, algunas reflexiones sobre las formas en las que el espacio afecta y/o es afectado por diversos procesos políticos o sociales, globales y regionales, nacionales y locales. Ciertamente, no existe un determinismo de la geografía o del medio, ya que el territorio forja y condiciona, pero no predetermina los resultados de la acción social. La sociedad construye y se apropia los espacios que permiten su reproducción y, al mismo tiempo, dichos espacios influyen en la agencia y en los procesos de cambio social. Vínculos de esta índole no son fáciles de analizar; requieren de herramientas teórico-metodológicas propicias para explicar por qué individuos con características sociodemográficas cuasiidénticas pueden comportarse de modo tan disímil en diferentes situaciones, contextos y lugares.

Con todo y los avances registrados, el análisis espacial sigue siendo incipiente en Latinoamérica y todavía carecemos de teorías integradas sobre el papel específico del territorio en la producción de los procesos sociopolíticos. Identificamos, así, una necesidad pendiente de estudios que permitan avanzar en este campo, con objetos analíticos y anclajes empíricos locales, nacionales y regionales. Agradecemos a América Latina Hoy la oportunidad y el espacio para seguir impulsando este enfoque en las ciencias sociales latinoamericanas.

\section{Bibliografía}

AGNEW, John A. Mapping politics: how context counts in electoral geography. Political Geography, 1996, vol. 15 (2): 129-146.

AnseLIN, Luc. Spatial Econometrics: Methods and Models. Dordrecht: Kluwer Academic Publishers, 1988.

BAsset, Yann. Balance electoral de Colombia 2010. Bogotá: Editorial Universidad del Rosario, 2011.

Blanquer, Jean-Michel; GIRALDO, Fernando y SOnNLeITnER, Willibald. Esbozo de geografía política de los países andinos: Hacia un Atlas electoral de América Latina. ALCEU, 2003, vol. 3 (6): 119-351.

Blanquer, Jean-Michel; Quanquin, Hélène; Sonnleitner, Willibald y Zumello, Christine. Voter dans les Amériques: Canada, États-Unis, Amérique latine. París: Éditions de l'Institut des Amériques, Instituto de Altos Estudios de América Latina-Universidad de París III, 2005.

BOn, Fréderic y ChEYlan, Jean-Paul. La France qui vote. París: Hachette (Pluriel), 1988.

Bussi, Michel. Eléments de géographie électorale à travers l'exemple de la France de l'Ouest. Rouen: Publications de l'Université de Rouen, n. 240, 1998. 
Calvo, Ernesto y Escolar, Marcelo. The Local Voter: A Geographicaly Weighted Approach to Ecological Inference. American Journal of Political Science, 2003, vol. 47 (1): 189-204.

Cox, Kevin R. The voting decision in a spatial context. Progress in Geography, 1969, vol. 1: 81-117.

EMmerich, Gustavo. Votos y Mapas. Estudios de geografía electoral en México. Toluca: UAM, 1993.

Escolar, Marcelo y Calvo, Ernesto. La nueva política de partidos en la Argentina. Buenos Aires: Prometeo, 2005.

EsCOLAR, Marcelo y CASTRO, Luis. Integración del sistema político y diferenciación geográfica del voto. El caso argentino 1983-1995-2007. Trabajo presentado en el VI Congreso Latinoamericano de Ciencia Política de la Asociación Latinoamericana de Ciencia Política (ALACIP). Quito, 12-14 de junio de 2012.

Goguel, François. Chroniques électorales. Paris: Presses de Sciences Po, 1981-1983.

GOGUEL, Francois. Géographie électorale et science politique: un itinéraire. Espaces Temps. Réfléchir les sciences sociales, 1990, vol. 43/44: 19-24.

Gómez TAgle, Silvia y VALDÉs, María Eugenia. La geografía del poder y las elecciones en México. México: Instituto Federal Electoral/Plaza y Valdés Vega, 2000.

Goodchild, Michael F. y Janelle, Donald G. Thinking Spatially in the Social Sciences. En Goodchild, Michael F. y JAnelle, Donald G. Spatially Integrated Social Sience. Oxford: Oxford University Press, 2004.

Goodchild, Michael F. y Janelle, Donald G. Toward critical spatial thinking in the social sciences and humanities. GeoJournal, 2010, vol. 75: 3-13.

JaCOB, Cesar Romero; HeEs, Dora Rodrigues; Waniez, Philippe y Brustlein, Violette. A geografia do voto nas eleições presidenciais do Brasil: 1989-2006. Río de Janeiro y São Paulo: PUC-Rio y Editora Vozes, 2010.

JoHnStOn, Ron. Political, Electoral and Spatial Systems. Oxford: Oxford University Press, 1979.

LACOSTE, Yves. Géopolitiques des régions francaises. París: Fayard, 1986.

LE BRAS, Hervé. Une autre France. París: Odile Jacob, 2002.

LE BRAS, Hervé y TodD, Emmanuel. L'invention de la France. París: Hachette, 1981.

LogAn, John R. Making a Place for Space: Spatial Thinking in Social Science. Annual Review of Sociology, 2012, vol. 38: 507-524.

Losada, Rodrigo; GIRALDO, Fernando y MuÑoz, Patricia. Atlas sobre las elecciones presidenciales de Colombia. 1974-2002. Bogotá: Pontificia Universidad Javeriana, 2004.

LSE. Charles Booth's London: Poverty maps and police notebooks, 2017, en línea: https://booth. lse.ac.uk. Fecha de consulta: 10 abril 2017.

Mayer, Nonna y Perrineau, Pascal. Les comportements politiques. París: Armand Colin, 1992.

Molinar Horcasitas, Juan. Geografía electoral. En MarTínez Assad, Carlos (coord.). Balance y perspectivas de los estudios regionales en México. México: CIIH-UnAm y Editorial Porrúa, 1991.

NorthwesternLaw. The Life and Times of Florence Kelley in Chicago, 1891-1899, 2017, en línea: http://florencekelley.northwestern.edu. Fecha de consulta: 10 abril 2017.

PARK, Robert E. y BuRGESS, Ernest. Introduction to the science of sociology. Chicago, Illinois: University of Chicago Press, 1921.

RoBINSON, William S. Ecological correlation and the behavior of individuals. American Sociological Review, 1950, vol. 15 (3): 351-357.

Romero Ballivián, Salvador. Geografía electoral de Bolivia. La Paz: Caraspas Fundemos, 2003.

Romero Ballivián, Salvador. Atlas Electoral Latinoamericano. La Paz: Corte Nacional Electoral, 2007. 
rsph. The John Snow Society, 2017, en línea: http://www.johnsnowsociety.org. Fecha de consulta: 10 abril 2017.

SIEGFrIED, André. Tableau politique de la France de l'Ouest. París: Librairie Armand Colin, 1913.

SOARES, Glémcio Ary Dillo y TERRON, Sonia Luiza. Dois Lulas: a geografia eleitoral da reeleição (explorando conceitos, métodos e técnicas de análise geoespacial). Opinião Pública, 2008, vol. 14 (2): 269-301.

SONNLEITNER, Willibald. Territorios y fronteras del voto: Hacia una agenda de geografía electoral para Centroamérica. TRACE. Travaux et Recherches dans les Amériques du Centre, 2005, vol. 48: 90-108.

SONNLEITNER, Willibald. Explorando los territorios del voto: Hacia un atlas electoral de Centroamérica. Guatemala: Centro de Estudios Mexicanos y Centroamericanos, Instituto de Altos Estudios de América Latina y Banco Interamericano de Desarrollo, 2006.

SONNLEITNER, Willibald. Explorando las dimensiones territoriales del comportamiento político: Reflexiones teórico-metodológicas sobre la geografía electoral, la cartografía exploratoria y los enfoques espaciales del voto. Estudios Sociológicos, 2013, vol. XXXI: 97-142.

TAYlor, Peter y House, John. Political Geography, Recent Advances and Future Directions. New Jersey: Barnes \& Noble Books, 1984.

TERron, Sonia Luiza. A Composição de Territórios Eleitorais no Brasil: Uma Análise das Votações de Lula (1989-2006). Tese de Doutorado. Río de Janeiro: IUPERJ, 2009.

TERron, Sonia Luiza y SOARES, Glémcio Ary Dillo. As bases eleitorais de Lula e do PT: do distanciamento ao divórcio. Opinião Pública, 2010, vol. 16 (2): 310-337.

Villalta Perdomo, Carlos. Sobre la espacialidad de los procesos electorales urbanos y una comparación entre las técnicas de regresión OLS y SAM. Estudios Demográficos y Urbanos, 2006, vol. 21 (1): 83-122.

WanIEZ, Philippe. Les données et le territoire au Brésil. Diplôme d'Habilitation à Diriger des Recherches en Géographie Humaine. Nanterre: Université Paris-X Nanterre, 2002.

WanIEZ, Philippe. Cartographie Thématique et analyse des données. Bordeaux: Université de Bordeaux, 2008.

Waniez, Philippe; Brustlein, Violette; JaCob, Cesar Romero; HeEs, Dora Rodrigues y De CaSTRO, Iná Elias. Différenciation spatiale et changements politiques, économiques et religieux au Brésil. En Lombard, J.; Mesclier, E. y Velut, S. (eds.). La Mondialisation côté Sud, Acteurs et territoires. París: IRD Éditions y ENS, 2006: 203-230.

Rodrigo RODRIGUES-SILVEIRA

Universidad de Salamanca

Sonia TERRON

Instituto Brasileiro de Geografia e Estatística

Willibald SONNLEITNER

El Colegio de México 